\title{
EVALUAR LA CALIDAD DE LOS PROGRAMAS DE EDUCACIÓN TEMPRANA A TRAVÉS DE UN PROTOCOLO. UN ESTUDIO DE CASO
}

Sonia Rivas Borrell

\section{RESUMEN}

Existe un interés creciente entre los investigadores para entender cómo se establece y se mantiene la calidad en los programas de educación temprana; cómo debe evaluarse, y cómo deben interpretarse los resultados obtenidos. Específicamente, en España, se reconoce la naturaleza educativa y asistencial de la etapa que comprende los primeros seis años de edad, en la que existen diversas propuestas pedagógicas de muy variada orientación. Ante la falta de instrumentos que ayuden a los centros a evaluar la calidad de los programas educativos, se describe el protocolo diseñado y aplicado para evaluar la calidad de un programa de educación temprana trilingüe (castellano, vascuence e inglés) en un centro del norte de España, durante dos cursos académicos.

Palabras clave: educación temprana; evaluación; protocolo; calidad. 


\section{ABSTRACT}

There is increasing interest amongst researchers for understanding how quality can be established and maintained in early childhood education, how it can be evaluated and how the results obtained should be interpreted. Specifically, Spanish legislation recognizes the educational and social nature of the stage comprised of the first six years for which there are numerous pedagogical proposals. Trying to face up to the lack of instruments to help evaluate the quality of these educational programs, we offer the results of the design and application of a protocol to evaluate the quality of a tri-lingual, early childhood educational program (Spanish, Basque and English) developed in a city in the North of Spain through two school years.

Key words: early childhood; assessment; protocol; quality.

\section{INTRODUCCIÓN}

Desde distintos ámbitos científicos — como la psicología del desarrollo, la neurociencia o la psicología infantil (De GraafPeters y HaddersAlgra, 2006; Lagercrantz, Hanson, Evrard y Rodeck, 2002; Volpe, 2001)_se apunta que el niño, durante los primeros años de vida, posee una gran capacidad para aprender. La aspiración de mejorar y desarrollar las habilidades infantiles, a través de intervenciones educativas llevadas a cabo tempranamente, se ha concretado ampliamente con éxito en el caso de actuaciones dirigidas hacia la población con discapacidad. Sin embargo, la finalidad de las actuaciones educativas dirigidas a la población sin discapacidad sigue siendo tema de intenso debate intelectual (David, 1990; Ferrandino, 2001; Knoche, Peterson, Edwards y Jeon, 2006). De momento, los investigadores mantienen cierta cautela a la hora de lanzar afirmaciones rotundas sobre la conveniencia de plantear a toda costa actividades que se consideran, coloquialmente hablando, «estimuladoras» para dicha población (Bruer, 2000, Little, 2001; Rivas, 2004a; Schiller, 2001; Shelden and Rush, 2001; Stover, 2001).

El motivo reside en que el desarrollo y la evaluación de las capacidades infantiles son temas de gran complejidad, por lo que no hay evidencia empírica de que las intervenciones educativas ofrecidas durante este 
período a la población sin discapacidad determinen sus habilidades futuras y su competencia de forma total o parcial (Shonkoff y Phillips, 2000; Slavenas, 2005), como se ha llegado a asegurar. De todos modos, esta premisa no debería llevarnos a limitar el desarrollo de programas educativos que puedan enriquecer el potencial del niño. Se defendería en este contexto el concepto de educación temprana y el de estimulación temprana, por ser éste:

[...] el proceso educativo global, secuencial, continuado y regulado, iniciado antes de los tres años de la vida del niño, aprovechando el momento en que el SNC se encuentra en el período de mayor plasticidad, y sin forzar su curso de desarrollo normal. Éste perseguiría, en el entorno escolar, la optimización y desarrollo de las capacidades del niño con o sin déficit presentes, para potenciar sus capacidades, siempre en estricta colaboración con el entorno familiar ${ }^{1}$.

El reconocimiento de que antes de los tres años se tiene una gran capacidad para aprender, se reconoce explícitamente en la legislación española cuando se habla de la naturaleza educativa, además de asistencial, de la etapa de escolaridad que abarca los tres primeros años de vida. Sin dejar de obviar que la dicotomía asistencial-educativa resulta peligrosa y ficticia en cierto sentido en esta etapa, en España se ha roto con la arraigada visión exclusivamente asistencial que siguen teniendo otros países. Si bien hasta hace relativamente pocos años la escolarización en los primeros años se había entendido como un servicio ofrecido a los padres como mera guarda y custodia de sus hijos, en España se considera que la escolarización, antes de los tres años de edad, cumple una doble finalidad: educativa y asistencial. Se conforman de este modo los seis primeros años de escolaridad como dos ciclos independientes de tres años de duración: primer ciclo de educación infantil (hasta los tres años) y segundo ciclo de educación infantil (desde los tres hasta los seis años). En ambos ciclos se apuesta por la aplicación de programas pedagógicos (LOE, 2006, título I, artículo 14, núm 3), y por la garantía de la calidad de las actuaciones educativas que se lleven a cabo (MEC, 2006).

${ }^{1}$ Sonia Rivas, Educación temprana en el niño de 0 a 3 años a través de programas, p. 102. 
Aunque en esta etapa se debe asegurar el cumplimiento de unos requisitos curriculares mínimos, que se traduce en un desarrollo de capacidades, los centros que ofertan estos ciclos cuentan con la libertad suficiente para aplicar el programa pedagógico que se adecue a su realidad educativa particular, siempre y cuando se respeten los mínimos acordados. En consecuencia, existe una variedad y riqueza de propuestas pedagógicas en los centros de educación infantil que contemplan el currículum desde muy distintas perspectivas, encontrándonos desde una perspectiva puramente lúdica hasta una perspectiva prioritariamente instructiva.

Como garante de un sistema educativo de calidad, la legislación española apuesta por la práctica evaluativa como la actividad obligatoria y necesaria en los centros educativos (LOE, 2006, título IV, artículo 140.a). Por ello, se deben someter a evaluación todos los elementos presentes en el proceso de enseñanza-aprendizaje. Sin embargo, no resulta tarea fácil llevar a cabo una evaluación en este primer tramo de escolaridad para garantizar la calidad de las intervenciones.

Se considera que la evaluación es todo aquel proceso sistemático, planificado y dirigido a identificar, obtener y analizar la información de cualquier acontecimiento educativo de una forma fiable y válida, tras una comparación con unos determinados parámetros de referencia, que sirvan de base para emitir un juicio de valor. Dicho juicio permite, a su vez, tomar decisiones para la mejora y optimización de cualquier elemento del proceso enseñanza-aprendizaje. La realidad educativa es cambiante y dinámica, de modo que pretender evaluar la calidad de un programa educativo desde una visión estática no aporta demasiada ayuda al educador.

Hay un interés creciente entre los investigadores para entender cómo se establece y se mantiene la calidad en los programas de educación temprana, cómo debe evaluarse y cómo deben interpretarse los resultados obtenidos (Clark y Stroud, 2002; Fontaine, Torre, Grafwallner y Underhill, 2006; Gol-Guben, 2007; Gullo, 2004; Lee y Walsh, 2004; Rivas, Sobrino y Peralta, 2005; Rivas, 2008; Sarancho y Spodek, 2007; Sheridan y Schuster, 2001). Como señala acertadamente 
Sarramona (2004), al hablar de calidad no se sabe exactamente a qué nos referimos si no se especifica el significado al que se hace referencia.

En educación, un criterio de calidad centrado exclusivamente en los resultados puede llevar a desvirtuar la naturaleza misma de la educación como una actividad valiosa por sí misma, de frutos siempre complejos y a largo plazo; pero es igualmente cierta la necesidad de incorporar las dimensiones cuantificables a la definición de calidad. Desde esta perspectiva, apenas existen guías o protocolos que, enriqueciéndose de la investigación aportada a este ámbito, iluminen al educador en su práctica diaria. En este sentido, es esencial contar con instrumentos que ayuden a evaluar la calidad de los programas en educación infantil.

Se han identificado diversos indicadores que se relacionan directamente con el criterio de calidad. Éstos agrupan la información de diversos modos, aunque la forma más conocida es la que divide los datos en variables estructurales y procesuales (Cryer, Tietze, Burchinal, 1999; Howes, Whitebook y Phillips, 1992; Phillips y cols., 2000; Rivas, 2004b). Mientras que las variables estructurales se refieren a elementos estáticos del programa (ratio adulto-niño, tamaño del grupo, o preparación del profesor, entre otras), las variables procesuales hacen alusión a factores dinámicos (actitud de los educadores durante la aplicación del programa o medidas de adecuación de la docencia). Si bien las variables estructurales pueden venir reguladas y definidas por la legislación vigente en el sistema educativo, dada su naturaleza estática, se necesita un análisis más complejo para identificar y regular la aplicación del segundo grupo de variables en la educación infantil.

No debemos pasar por alto que sería una simplificación limitar la preocupación sobre la evaluación de la calidad de programas en hallar indicadores que arrojen luz sobre el proceso evaluador. Sin embargo, partiendo de que no debe reducirse la evaluación a este punto (Lee y Walsh, 2004; Rivas, Sobrino y Peralta, 2005), es vital encontrar indicadores que ayuden al profesional a mejorar la calidad de sus intervenciones desde una aproximación sistemática, así como a establecer principios que auxilien a los profesionales a estar de acuerdo en los mismos criterios en esta etapa tan compleja de delimitar (Rivas, 2004b). 


\section{OBJETIVO}

El objetivo de este escrito es mostrar el resultado del diseño de un protocolo para evaluar la calidad de un programa de educación temprana trilingüe (castellano, vascuence e inglés) desarrollado en una ciudad del norte de España, y los resultados de su aplicación durante dos cursos académicos (1999-2000 y 2000-2001) para conocer su resultado.

\section{DESCRIPCIÓN DEL CENTRO Y DEL PROGRAMA}

El programa sobre el que se diseña y aplica el protocolo de evaluación de la calidad se denomina Programa de estimulación temprana y trilingüismo. Se trata de un centro privado de orientación católica, situado en la ciudad del norte de España de San Sebastián, que da servicio a alrededor de 50 familias de clase social medio-alta. Cuenta con unas instalaciones amplias, con zona ajardinada propia y un servicio completo de comedor. Dicho programa funciona de lunes a viernes durante siete horas y media al día. De modo opcional, el centro ofrece un servicio de guarda y custodia de los niños que lo requieran, los sábados por la mañana.

Los profesionales que trabajan en el centro ( $100 \%$ femenino) proceden de diversos niveles educativos, con preparación pedagógica adecuada y con dominio certificable de las tres lenguas del programa: inglés (nivel Proficiency), español (licenciatura hispánica o similar) y vasco (título de EGA). El rango de edad del profesorado oscila entre los 23 y los 33 años de edad. En cada clase hay una ratio de 11 niños por cada 2 profesoras, independientemente de la actividad que se acometa en cada grupo y en cada momento. Así se respeta la ratio legal de 1:8 sugerida en la legislación española para ese período.

El programa tiene una fuerte connotación académico-instructiva, y se centra en el desarrollo de las capacidades sociales, cognitivas, motrices y lingüísticas, haciendo especial hincapié en las dos últimas.

La metodología del programa pivota en el desarrollo de las competencias lingüísticas del niño. Los docentes hablan de «inmersión lingüística», evocando la imagen de sumergir al niño en un entorno en donde todo fomente el aprendizaje de distintas lenguas. Así, cada 
profesora utiliza un único idioma a lo largo de su jornada laboral en todas las actividades que realice y en todos los lugares en los que ésta se mueva: desde el comedor, hasta la clase, pasando por las horas de descanso. La docente realiza tareas en conjunto con el grupo de clase durante 20 minutos, junto con otra profesora que habla otro idioma distinto al suyo. Así, el niño recibe ante las mismas situaciones de aprendizaje y de clase los inputs de dos profesoras que hablan dos idiomas diferentes, realizando la tarea que corresponda en ese momento. Mientras una docente ejerce la función de profesora principal, la otra realiza una labor de apoyo. Como la metodología del programa se fundamenta en la rotación del profesorado, a los 20 minutos, otras dos profesoras distintas entran en el aula siguiendo la misma dinámica. De este modo, a lo largo del día y en una misma aula el alumno habrá estado en contacto con varias profesoras que se habrán comunicado con él en distintos idiomas, en las actividades previstas en el horario.

El área motora se aborda a través de actividades con denominación propias como el «gateo», el «arrastre», la «braquiación» o la «croqueta». El programa enumera así diversos ejercicios de psicomotricidad que encuentran reminiscencias en la teoría del desarrollo cerebral de Doman (1993), puestas en tela de juicio en numerosas ocasiones (American Academy of Pediatrics, 1982, 1999).

El desarrollo del área cognitivo-social se aborda con un material pedagógico ya de uso conocido: los denominados bits de inteligencia, empleados principalmente para favorecer la transmisión de contenido a través de imágenes. La estructuración y la secuenciación de las actividades del programa favorecen que los niños conozcan así distintos términos que, por descubrimiento o en otro contexto, sería complejo conocer. A través de los bits se les muestra la imagen de las diferentes obras de arte universalmente conocidas, o la rosa de los vientos por ejemplo, contenido que han memorizado aprovechando sus recorridos habituales por el centro, donde está distribuido dicho material.

Según las bases del programa, las actividades descritas favorecen el correcto desarrollo del sistema nervioso central del niño, por la trascendencia que supone ofrecerle ambientes enriquecidos y estimulantes 
durante las primeras edades para la estabilización sináptica. Por este motivo, se justifica la carga de contenido conceptual y la metodología del programa. También se defiende el fundamento antropológico, en cuanto que se considera que cada alumno es único, por lo que deben favorecerse sus propios intereses, preferencias y capacidades. Asimismo, se habla de bases pedagógicas cuando señalan que el niño debe estar inmerso en un ambiente que le ofrezca la oportunidad para aprender y desarrollar todas las capacidades posibles. Finalmente, las bases psicológicas se evidencian en el programa cuando se habla de la individualización de la enseñanza que favorecen los docentes.

\section{RESULTADOS}

Hacen referencia a dos aspectos diferenciados: al diseño del protocolo como instrumento de evaluación, y a la aplicación de dicho protocolo.

\section{Resultados sobre el Diseño del Protocolo}

El protocolo diseñado se sustenta en el modelo de evaluación de Pérez Juste (1995), en la teoría de Glassman y Nevo (1988), y en la propuesta de Román y Díez (1994). La información ofrecida aborda cuestiones desde distintas perspectivas con el fin de determinar la calidad del programa desde todos sus ángulos. El protocolo diferencia datos sobre el programa en sí mismo (la calidad que tiene el programa en sí, independientemente de su puesta en marcha), sobre el programa en su proceso (información relativa a las características del programa durante su implantación) y sobre el programa en sus resultados (datos sobre la eficacia del programa). La recogida de información se realiza por un único evaluador experto en tres momentos temporales distintos: antes de la aplicación del programa, durante la ejecución del mismo y en su finalización.

El protocolo diseñado para evaluar la calidad del programa se organiza en cinco grandes áreas: (1) alumnos, (2) profesores, (3) padres, (4) datos del contexto educativo, y (5) documentación del programa.

A cada agente se le plantea una pregunta relacionada con la calidad de la intervención. Dicha pregunta, a su vez, se concreta en distintas 
dimensiones, plasmadas en diversos indicadores que, a su vez, se traducen en diferentes variables (Cfr. Tabla 1 ).

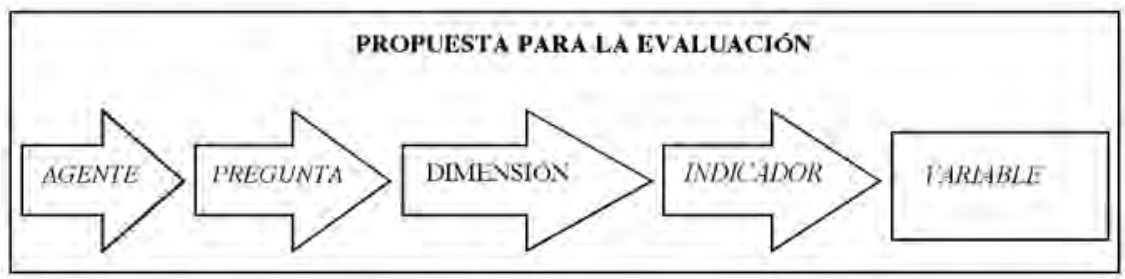

Tabla 1. Propuesta para la evaluación, adaptada de Román y Diez (1994).

El protocolo se conforma por trece columnas, donde se combina la información recogida de los diferentes agentes implicados en la evaluación de este programa, así como del contexto y del documento del programa, en tres momentos distintos en los que ésta se ha llevado a cabo (antes, durante y después de la aplicación del programa) (Rivas, 2008). Como resultado, se obtiene una visión de conjunto sobre el proceso de la evaluación realizada (quién es el agente principal, limitaciones encontradas, valoración de la variable, instrumento aplicado, fecha de aplicación y definición según el modelo de evaluación elegido), así como información detallada sobre la calidad del programa en sí mismo, en su proceso y en sus resultados.

Las variables de las que se recoge información refieren a los siguientes agentes: profesores, padres, alumnos, así como información sobre el contexto y sobre el documento del programa.

\subsection{Variables relativas a los profesores}

La calidad de las prácticas y las actitudes del profesorado son un componente crucial a la hora de definir las intervenciones educativas de calidad. Existe todo un cuerpo teórico que avala la relación entre la calidad de los programas y la capacitación profesional del educador (Blau, 2000; Buysse, Wesley, Bryant y Gardner, 1999). Por esta razón, en el protocolo se distinguen tres dimensiones que identifican la calidad del programa con la figura del educador: su formación académica, sus actitudes y su experiencia práctica. 
Las actitudes del docente se valoran a partir de tres indicadores: la actitud entre los docentes, la actitud de los educadores hacia los padres y su actitud hacia el programa. La práctica educativa, en cambio, se analiza valorando el clima mantenido en clase y los recursos evaluativos empleados por los docentes (Cfr. Tabla 2).

\begin{tabular}{|c|c|c|c|}
\hline AGENTE & DIMENSIÓN & INDICADOR & VARIABLE \\
\hline \multirow{20}{*}{ PROFESOR } & \multirow[t]{6}{*}{ Formación. } & \multirow[t]{2}{*}{ Preparación didáctica. } & $\begin{array}{l}\text { Años de experiencia en el } \\
\text { centro. }\end{array}$ \\
\hline & & & $\begin{array}{l}\text { Años de experiencia en el } \\
\text { ámbito educativo. }\end{array}$ \\
\hline & & \multirow{2}{*}{ Formación pedagógica. } & $\begin{array}{l}\text { Estudios de posgrado y } \\
\text { estancias en el extranjero. }\end{array}$ \\
\hline & & & $\begin{array}{l}\text { Conocimiento y dominio de } \\
\text { idiomas. }\end{array}$ \\
\hline & & \multirow{2}{*}{$\begin{array}{l}\text { Actualización pedagógica } \\
\text { y formación continua. }\end{array}$} & $\begin{array}{l}\text { Formación previa sobre el } \\
\text { programa. }\end{array}$ \\
\hline & & & $\begin{array}{l}\text { Formación en otros temas } \\
\text { educativos. }\end{array}$ \\
\hline & \multirow{10}{*}{$\begin{array}{l}\text { Practica } \\
\text { docente. }\end{array}$} & ...entre las docentes. & ...dentro y fuera del aula. \\
\hline & & \multirow[b]{2}{*}{...hacia los padres. } & en el hall de la escuela. \\
\hline & & & en otros momentos. \\
\hline & & \multirow[b]{2}{*}{...hacia el programa. } & Respecto a la metodología. \\
\hline & & & $\begin{array}{l}\text { Respecto a los contenidos, } \\
\text { actividades y evaluación. }\end{array}$ \\
\hline & & \multirow{5}{*}{ Clima de clase. } & Tiempo de comidas. \\
\hline & & & Tiempo de descanso y lúdico. \\
\hline & & & Tiempo de higiene. \\
\hline & & & Entrega a los padres. \\
\hline & & & Tiempo curricular. \\
\hline & & \multirow{4}{*}{ Practica evaluativo. } & Objeto de la evaluación. \\
\hline & & & Momento de la evaluación. \\
\hline & & & $\begin{array}{l}\text { Frecuencia de } \\
\text { la evaluación. }\end{array}$ \\
\hline & & & $\begin{array}{l}\text { Tipo de contacto con los } \\
\text { padres. }\end{array}$ \\
\hline
\end{tabular}




\subsection{Variables relativas a los padres}

Los padres son copartícipes en la educación del niño junto con el educador, en cuanto le han dejado encomendada la labor asistencial y educativa. En efecto, las familias no solamente representan, además de sus alumnos, los destinatarios próximos de la actuación de la escuela, sino que son agentes condicionantes de la efectividad de la educación escolar. En este sentido, los programas de calidad en educación infantil se caracterizan por el alto grado de satisfacción parental, fruto del buen nivel de comunicación e intercambio de información entre las familias y el centro escolar (Cryer y Burchinal, 2003; Rivas, 2007a, 2007b). El grado de participación e implicación de los padres en la escuela también se ha identificado como un factor de calidad (Richard, Minish y Zhou, 1993; Castellanos, 2000), siendo definido incluso como el factor determinante para valorar la calidad de los programas (Beckman, Robinson, Rosenberg y Filer, 1994; Reynolds, Mavrogenes, Bezruczko y Hagemann, 1996). Por esta razón, el protocolo toma en consideración dos dimensiones diferenciadas en la investigación: la satisfacción parental y el grado de implicación de las familias en la escuela (Cfr. Tabla 3).

\begin{tabular}{|c|c|c|c|c|}
\hline AGENTE & OBJETIVO & DIMENSIÓN & INDICADOR & VARIABLE \\
\hline \multirow{6}{*}{ PROFESOR } & \multirow{6}{*}{$\begin{array}{l}\text { Grado de } \\
\text { satisfacción } \\
\text { con el } \\
\text { programa. }\end{array}$} & \multirow{4}{*}{ Satisfacción. } & Con el personal. & $\begin{array}{l}\text { Por el trato ofrecido a su hijo. } \\
\text { Por el trato ofrecido hacia ellos. }\end{array}$ \\
\hline & & & Con las instalaciones. & Con el jardín, comedor y aulas. \\
\hline & & & \multirow{2}{*}{ Con el programa. } & En su vertiente asistencial. \\
\hline & & & & En su vertiente educativa. \\
\hline & & \multirow{2}{*}{ Implicación. } & Con la escuela. & $\begin{array}{l}\text { Canales de comunicación } \\
\text { empleados. }\end{array}$ \\
\hline & & & $\begin{array}{l}\text { Con su formación } \\
\text { como padres. }\end{array}$ & $\begin{array}{l}\text { Participación en } \\
\text { la Escuela de Padres. }\end{array}$ \\
\hline
\end{tabular}

Tabla 3. Parte del protocolo correspondiente al agente padres. 


\subsection{Variables relativas a los alumnos}

Dado que programa de educación temprana trilingüe fomenta el desarrollo de distintas capacidades (cognitivas, motrices, de comunicación y de socialización), el protocolo analiza los resultados referidos a cada una de ellas (Cfr. Tabla 4). Como las capacidades en sí mismas no pueden ser analizadas por su alto grado de generalidad, se someten a evaluación las habilidades que presenta el niño ante distintas tareas que son, a su vez, la concreción de las destrezas que provienen de estas capacidades. Este objetivo se logra gracias a la aplicación de la escala Haizea (Fuentes-Biggi y Fernández, 1992), instrumento de conocida reputación en España entre los profesionales de atención temprana (Llanos y Azurmendi, 2002; Iceta y Yoldi, 2002).

\begin{tabular}{|c|c|c|c|c|}
\hline AGENTE & OBJETIVO & DIMENSIÓN & INDICADOR & VARIABLE \\
\hline \multirow{7}{*}{ ALUMNO } & \multirow{7}{*}{$\begin{array}{l}\text { Nivel de } \\
\text { desarrollo } \\
\text { adquirido } \\
\text { tras el } \\
\text { programa. }\end{array}$} & \multirow{5}{*}{ Capacidades. } & \multirow{2}{*}{$\begin{array}{l}\text { Habilidades } \\
\text { de socialización. }\end{array}$} & Socialización. \\
\hline & & & & Autonomía. \\
\hline & & & $\begin{array}{l}\text { Habilidades } \\
\text { lógicas. }\end{array}$ & Lógico-matemática. \\
\hline & & & \multirow{2}{*}{$\begin{array}{l}\text { Habilidades } \\
\text { motrices. }\end{array}$} & Motricidad gruesa. \\
\hline & & & & Motricidad fina. \\
\hline & & & $\begin{array}{l}\text { Habilidades } \\
\text { comunicativas. }\end{array}$ & Comunicación expresiva. \\
\hline & & & $\begin{array}{l}\text { Con su formación } \\
\text { como padres. }\end{array}$ & Comunicación receptiva. \\
\hline
\end{tabular}

Tabla 4. Parte del protocolo correspondiente al agente alumno.

\subsection{Variables relativas al contexto}

El contexto se entiende como el medio físico, el entorno en el que se desarrolla el programa. Es notablemente aceptado que la disposición del contexto físico influye en los resultados del programa y en la calidad del 
mismo. Por esta razón, el protocolo recoge el marco físico a partir de unas dimensiones determinadas, extraídas del instrumento empleado para valorarlo: la versión española de la escala ECERS (Palacios y Lera, 1992), instrumento que recoge información sobre la calidad de los contextos educativos. Ésta contiene las siguientes subescalas: (1) rutinas de cuidado personal de los niños, (2) mobiliario para los niños y materiales disponibles para su aprendizaje, (3) experiencias de lenguaje y razonamiento, (4) actividades de motricidad gruesa, (5) actividades creativas, (6) desarrollo social, y (7) equipamiento para las necesidades del adulto. En total, la escala se compone de 37 items, que son puntuados entre 7 (valoración excelente) y 1 punto (valoración inadecuada).

\subsection{Variables relativas al documento del programa}

El protocolo da cuenta de la calidad de las bases científicas del programa a partir de dos dimensiones: el marco neurológico y el marco psicológico sobre el que se apoya (Cfr. Tabla 5). Para tal fin se toman en consideración preguntas del modelo de Pérez Juste (1995) relativas al contenido del programa.

\begin{tabular}{|l|l|l|l|l|}
\hline AGENTE & OBJETIV0 & DIMENSIÓN & \multicolumn{1}{|c|}{ INDICADOR } & \multicolumn{1}{c|}{ VARIABLE } \\
\hline \multirow{2}{*}{ PROGRAMA } & $\begin{array}{l}\text { Calidad } \\
\text { del } \\
\text { documento. }\end{array}$ & $\begin{array}{l}\text { Calidad } \\
\text { científica. }\end{array}$ & $\begin{array}{l}\text { Marco } \\
\text { neurológico. }\end{array}$ & $\begin{array}{l}\text { Explicitación de } \\
\text { las bases científicas. }\end{array}$ \\
\cline { 3 - 5 } & & $\begin{array}{l}\text { Calidad } \\
\text { técnica. }\end{array}$ & Marco psicológico. & $\begin{array}{l}\text { Relevancia desde la pers- } \\
\text { pectiva científica. }\end{array}$ \\
\cline { 3 - 5 } & & $\begin{array}{l}\text { Inclusión de objetivos, } \\
\text { actividades, medios y } \\
\text { evaluación. }\end{array}$ \\
\cline { 3 - 5 } & & $\begin{array}{l}\text { Adecuación de los } \\
\text { objetivos, actividades y } \\
\text { sistemas de evaluación. }\end{array}$ \\
\hline
\end{tabular}

Tabla 5. Parte del protocolo correspondiente al agente programa. 


\section{Resultados Relativos a la Aplicación del Protocolo}

Se parte de la premisa que cualquier toma de decisiones en la evaluación del programa se orienta hacia la mejora del mismo, y no hacia su sanción. Así, los resultados obtenidos con este protocolo apuntan lo siguiente. Por lo que hace referencia al programa en sí mismo se detectan puntos fuertes y débiles, óptimos y mejorables en él. Como puntos débiles o mejorables del programa en sí, cabe referirse a que el marco neurológico de este programa no se sustenta sobre unas bases lo suficientemente sólidas como para justificar sus actividades. Las bases sobre las que se justifica el presente programa tienen reminiscencias claras del método Doman, el cual recibió durísimas críticas, entre otros motivos, por su falta de cientificidad, rigurosidad, por apoyarse en premisas falsas, y porque el análisis científico del mismo ha sido blindado (Glass y Robbins, 1967). Ha sido muy controvertido y criticado por autores de notable relevancia como Cummins (1988, 1992), Glass (1967), Robbins (1965), Gold y Kline (1985), e incluso por alguna institución de gran prestigio como la American Academy of Pediatrics (1982, 1999). Por este motivo, los fundamentos neurológicos del programa evaluado son un punto débil del mismo.

El marco psicológico, por otro lado, no responde a los principios que se sugieren en España para educación infantil (principio de globalización de las actividades y de individualización de la enseñanza).

En cambio, un punto fuerte del programa en sí mismo es su calidad técnica: la inclusión de objetivos, actividades, medios y sistemas de evaluación en el programa; y la adecuación de los objetivos, actividades, medios y sistemas de evaluación de enseñanza, es muestra de ello. Además, se detecta que el funcionamiento participativo del profesorado, el trabajo en equipo entre los docentes y los miembros de dirección puede considerarse un aspecto muy loable que podría justificar los buenos resultados obtenidos, por consiguiente, la eficacia, y con ella, la calidad (Rivas y Sobrino, en prensa).

La evaluación de este programa revela que los padres se sienten plenamente satisfechos con la parte educativa de este programa, a lo que se debe sumar la satisfacción por la atención asistencial dispensada. Es decir, que se considere este tramo de edad como un momento para propiciar el 
aprendizaje del niño no debe dejar de lado que la calidad asistencial (alimentación e higiene) es parte fundamental y definitoria de este primer tramo, ahora también educativo, que los padres exigen. El sentimiento de intranquilidad de las familias al tener que delegar en la escuela la función formativa y asistencial de los hijos se subsana, en gran parte, estrechando los lazos de comunicación entre el centro y la familia. Precisamente, estas vías de comunicación han sido una variable por la cual todos los padres han manifestado gran satisfacción y se plantean como futuras líneas de investigación.

El programa en su desarrollo, que incluye la ejecución y el marco del programa, son elementos que constituyen un punto fuerte, eficaz, del presente trabajo. De esta premisa cabría deducir que la función del educador es un factor esencial, clave y fundamental en cualquier programa para lograr unos buenos resultados. Su altísima preparación didáctica, formación pedagógica y formación continua han sido variables que se han acompañado de una alta motivación ante el trabajo y de un trabajo en equipo bien coordinado. En alguna medida, cabe sospechar que la confluencia de todas estas variables en la aplicación del programa podría explicar los buenos logros conseguidos con este programa, sobre todo si se tiene en cuenta su baja calidad científica.

El programa en sus resultados manifiesta que éste, siguiendo esta metodología y con este contexto determinado, contribuye al desarrollo social, motriz, cognitivo y lingüístico de los niños entre dos y tres años de edad. Sin embargo, cabe apuntar que la intensidad, duración y dedicación de este programa son superiores a los de otras intervenciones en infantil, si bien los resultados a los que llega este programa pueden ser similares a los que obtienen a través de otros programas.

\section{CONCLUSIONES}

El evaluar las intervenciones educativas es un modo de asegurar y garantizar su calidad, principio que en la legislación española - aunque extrapolable a otros contextos- constituye tanto una necesidad como una obligación. Valorar sistemáticamente las intervenciones es el único 
modo, dada la autonomía pedagógica de la que goza el profesor en estos primeros años de escolaridad, de verificar que el proceso de enseñanzaaprendizaje que se sigue en el centro es eficaz y que contribuye a la consecución de los objetivos propuestos. Por esta razón, se plantea también como una necesidad. Ésta abriría paso a la obligatoriedad en cuanto que la legislación sustenta que deben someterse a evaluación todos los elementos del proceso enseñanza-aprendizaje con el fin de garantizar un nivel de calidad aceptable.

Que sea una necesidad y una obligación no implica que resulte una tarea fácil: en primer lugar, es complejo encontrar criterios válidos y fiables en este primer ciclo, con una nomenclatura tan amplia como programas de educación infantil, que han englobado actuaciones educativas poco homogéneas; en segundo lugar, es difícil llegar a conclusiones que no distorsionen la realidad, porque con los resultados del programa se pueden establecer relaciones no reales o erróneas; en tercer lugar, la evaluación no puede desentenderse del contexto donde se desarrolla, pero controlar sus variables es una tarea casi imposible; en cuarto lugar, se corre el peligro de extraer conclusiones equivocadas o imprecisas de las herramientas o instrumentos que se empleen para obtener información sobre el programa. De todos modos, a pesar de éstas y otras dificultades que pueden plantearse a la hora de evaluar un programa de educación infantil que aluden al análisis y generalización de los resultados (limitaciones de orden técnico, moral y ambiental, además de los límites derivados del propio objeto) (Rivas, Sobrino y Peralta, en prensa), se cuenta con ellas para seguir adelante.

En este contexto se puede plantear el protocolo como herramienta de ayuda al profesional en este proceso evaluativo. Sin embargo, el protocolo como tal no debería convertirse en un absoluto; debería ser visto más bien como un trabajo en progreso, como una ayuda que se va mejorando con la investigación. En cualquier caso, el protocolo diseñado y aplicado puede ser el primer paso para una pertinente toma de decisiones sobre la mejora del programa en cualquiera de sus partes (en sí mismo, en su proceso y en su producto). El fin no se orienta a la reprobación, sino a ser consciente de los aspectos menos positivos o más débiles, para mejorarlos; y los más fuertes o positivos para subrayarlos, porque un buen 
sistema de evaluación de un programa debe ofrecer información útil para tomar decisiones para la mejora.

En este punto cabría hacer un punto de inflexión deteniéndonos en el contenido que ha evaluado el presente protocolo. No cabe duda de que llegaríamos a un consenso entre los educadores de educación infantil, tanto en España como en el resto de los países, si afirmamos que en educación infantil se trata de promover un desarrollo completo e integral del niño. Sin embargo, no habría el mismo acuerdo a la hora de admitir el tipo de contenido y metodología, y en menor medida en saber el grado de calidad de los programas. Dado el alto número de centros que ofertan o pueden ofertar estos primeros años de escolaridad, la variedad de contextos y la alta autonomía del profesor a la hora de diseñar sus intervenciones, se debe hacer un gran esfuerzo a la hora de entender qué es lo mejor para el niño y qué actividades conducen a ello. Al menos en España, se vive un momento de cierta presión social, pedagógicamente hablando, de corrientes opuestas que tratan de promover distintos curricula y metodologías en educación infantil: por un lado, se defienden programas con un alto carácter instructivo para que el niño esté preparado, listo para afrontar aprendizajes futuros; por otro lado, en cambio, se alzan voces que se decantan por el polo opuesto.

La preparación escolar en la infancia debe suponer que el aprendizaje sea el apropiado al desarrollo del niño, tanto en cuanto a sus peculiaridades contextuales, como a sus necesidades psicológicas y pedagógicas. En este sentido, los programas de educación temprana, sean cuales sean, debería evitar pensar en un producto dirigido más hacia el agrado de los padres (por lo brillantes que pueden parecer sus hijos en la escuela que sigue determinado programa, por la alta preparación con la que salen sus hijos en este mundo tan competitivo, por la cultura de la prisa que nos persigue y que hace que cada vez adelantemos más lo que nos corresponde aprender a cada edad...) que en las necesidades reales del niño, premisa que no implicaría ralentizar su aprendizaje. En este sentido, el manejo de los tiempos y los métodos de enseñanza en educación infantil debería fundamentarse en la consideración de la infancia como un período único, propio e irrepetible, en vez de ser una réplica pedagógica de ciclos superiores, por el peligro que ello conlleva. Si bien el día a día muestra cómo 
los niños son capaces de aprender a través de diversas metodologías, quizás deberíamos ser conscientes de la cantidad de contenido que se transmite con los programas de educación temprana. Puede ser tan nociva la hipoestimulación como la hiperestimulación durante la infancia, que podría llegar a ser contraproducente tanto para el niño (promoviendo conductas de falta de atención y de concentración) como para el educador (convirtiéndole, más que en un educador, en un «aplicador» de ejercicios pautados). Por ello, llegar a un consenso sobre qué constituye y qué aborda un programa de calidad en educación infantil, y cómo evaluarlo, se sigue planteando como un reto y como una necesidad imperiosa.

\section{BIBLIOGRAFÍA}

AMERICAN ACADEMY OF PEDIATRICS, «Position statement on Doman Delacato treatments of neurologically handicapped children», Pediatrics, 70 (5), 1982, p. 810-812.

AMERICAN ACADEMY OF PEDIATRICS, «The treatment of Neurologically impaired children using patterning», Pediatrics, 1999, 104 (5), p. 1149-1151.

BECKMAN, P.J.; ROBINSON, C.C.; ROSENBERG, S. \& FILER, J., «Family involvement in early intervention: the evolution of family centered service», en Johnson L.J.; Gallagher, R.J.; LaMontagne, M.J.; Jordan, J.B.; Gallagher, J.J.; Hutinger, P.L. \& Karnes, M.B. (eds.), Meeting early intervention changes. Issues from birth to three, Paul H. Brookes Publishing Co., Baltimore, 1994, p. 13-32.

BLAU, D.M., «The effect of child care characteristics on child development», The Journal of Human Resources, 29 (4), 2000, p. 786-822.

BRUER, J.T., El mito de los tres primeros años. Una nueva visión del desarrollo inicial del cerebro y del aprendizaje a lo largo de la vida, Paidós, Barcelona, 2000.

BURCHINAL, M. \& CRYER, D., «Diversity, child care quality, and developmental outcomes», Early Childhood Research Quarterly, XVIII, 2003, p. 401-426. 
BUYSSE, V.; WESLEY, P.W.; BRYANT, D. \& GARDNER, D., «Quality of early childhood programs in inclusive and noninclusive settings», Exceptional Children, LXV (3), 1999, p. 301-314.

CASTELLANOS, G., «La orientación familiar como experiencia pedagógica», Revista Panamericana de Pedagogía, 1, 2000, p. 39-55.

CLARK, P. \& STROUD, J., «Working Together to Improve the Quality of Care and Education in Early Childhood Programs», Early Child Development and Care, 172, 2000, p. 55-63.

CRYER, D.; TIETZE, W.; BURCHINAL, M.; LERA, M.J. \& PALACIOS, J., «Predicting process quality from structural quality in preschool programs: a cross country comparison», Early Childhood Research Quarterly, XIV: 3, 1999, p. 339-361.

DAVID, T., Under five, under-educated? Open University Press, Buckingham, 1990.

DE GRAAF-PETERS \& HADDERS-ALGRA, M., "Ontogeny of the human central nervous system: What is happening when?», Early Human Development, 82 (4), 2006, p. 257-266.

FERRANDINO, V., «Never too young to learn», Principal, 80, 2001, p. 64.

FONTAINE, N.S.; TORRE, L.D.; GRAFWALLNER, R. \& UNDERHILL, B., «Increasing quality in early care and learning environments», 176, 2006, p. 157-169.

FUENTES-BIGGI, J. y FERNÁNDEZ ÁLVAREZ, E., Escalas HaizeaLlevant para la evaluación del desarrollo de 0 a 6 años, Gobierno Vasco y Generalitat de Cataluña, San Sebastián, 1992.

GENTO, S., Instituciones educativas para la calidad total, La Muralla, Madrid, 2002.

GLASS, G. \& ROBBINS, M.P., A critique of experiments on the role of neurological organization in reading performance, University of Colorado, 1967.

GOLD, S. \& KLINE, J., «No miracles», Academic Therapy, 21 (2), 1985, p. 177-202.

GOL-GUBEN, M., «Evaluation of the quality of early childhood classrooms in Turkey», Early Child Development and Care, (en prensa). GULLO, D., Understanding assessment and evaluation in early childhood education, $2^{a}$ ed., Teachers College Press, New York, 2004. HOWES, C.; PHILLIPS, D. \& WHITEBOOK, M., «Threshold of quality: 
implications for the social development of children in center-based child care», Child Development, LXIII, 1992, p. 449-460.

KNOCHE, L.; PETERSON, C.A.; POPE, C. \& HYUN-JOO, E., "Childcare for children with and without disabilities: the provider, observer and parent perspectives», Early Childhood Research Quarterly, 21 (1), 2006, p. 93-109.

LAGERCRANTZ, H.; HANSON, H.; EVRARD, P. \& RODECK, C., The newborn brain. Neuroscience and clinical applications, Cambridge University Press, Cambridge, 2002.

LEE, J.H. \& WALSH, D.J., "Quality in early childhood programs: reflections from program evaluation practices», American Journal of Evaluation, 25, 2004, p. 351-373.

LITTLE, C.A., «The myth of the first three years: a new understanding of early brain development and lifelong learning», The Gifted Child Quarterly, 45 (3), 2001, p. 226-227.

LOE., Ley Orgánica 2/2006, de 3 de mayo, de Educación, BOE, 4-V2006.

MEC., «Evaluaciones generales del sistema educativo español», Apuntes del Instituto de Evaluación [en línea], núm. 8, 20-V-2008, en: [http: / / www.institutodeevaluacion.mec.es / contenidos/apuntes / ap untesn82006.pdf].

NEVO, C., Evaluation in decision making: the case of school administration, Kluwer, Boston, 1988.

PALACIOS, J. y LERA, M.J., Escala de valoración del entorno educativo, Traducción de ECERS, Visor, Madrid, 1992.

PÉREZ JUSTE, R., «Evaluación interna de programas educativos», p. 131-168, en Pérez Juste, R.; García, J.L. y Martínez, C. (eds.), Evaluación de programas y centros educativos, UNED, Madrid, 1995. PHILLIPS, D.; MEKOS, D.; SCARR, S.; MCCARTNEY, K. \& ABBOTSHIM, M., «Within and beyond the classroom door: assessing quality in child care centres», Early Childhood Research Quarterly, XV (4), 2000, p. 475-496.

REYNOLDS, A.J.; MAVROGENES, N.A.; BEZRUCZKO, N. \& HAGEMANN, M., "Cognitive and family support mediators of preschool effectiveness: a confirmatory analysis», Child Development, 67, 1996, p. 1119-1140. 
RICHARD, E.; MINISH, P.A. \& ZHOU, Q., «Parent involvement and quality day care in proprietary centers», Journal of Research in Childhood Education, 7, 1993, p. 53-61.

RIVAS, S., Educación temprana en el niño de 0 a 3 años a través de programas, EUNSA, Pamplona, 2004a.

, «Los indicadores de calidad en los programas de educación preescolar: elementos de juicio para los padres y educadores», Estudios Sobre Educación, 6, 2004b, p. 86-104.

, «Entender el ejercicio de la participación en padres y profesores de infantil: los padres y los educadores: un nuevo reto educativo», International Journal of Developmental and Educational Psychology, 1(2), 2007a, p. 323-334.

, «La participación de las familias en la escuela», Revista Española de Pedagogía, 238, 2007b, p. 39-56.

Evaluación de la calidad de un programa de educación temprana trilingüe para niños menores de tres años, comunicación presentada en el V Congreso Internacional de Psicología y Educación organizado por la Asociación de Psicología Evolutiva y de la Educación de la Universidad de Oviedo, España, 23 al 25 de abril, 2008.

RIVAS, S. \& SOBRINO, A., «The Design and application of a protocol for determining quality of infant-toddler programs in Spain: A case study», Early Years, (en prensa).

RIVAS, S.; SOBRINO, A. y PERALTA, F., «La evaluación como garantía de calidad en educación preescolar», Revista Española de Pedagogía, 63 (232), 2005, p. 511-528.

ROMÁN, M. y DÍEZ, E., Currículum y enseñanza. Una didáctica centrada en procesos, EOS, Madrid, 1994.

SARANCHO, O. y SPODEK, B., «Early childhood teachers' preparation and the quality of program outcomes», Early Child Development and Care, 177 (1), 2007, p. 71-91.

SARRAMONA, J., Factores e indicadores de calidad en la educación, Octaedro, Barcelona, 2004.

SCHILLER, P.B., «Brain research: its implications for early childhood programs», Child Care Inform Exchange, 140, 2001, p. 14-18.

SHELDEN, M.L. \& RUSH, D.D., «The ten myths about providing early intervention services in natural environments», Infants and Young Children, 14 (1), 2001, p. 1-13. 
SHERIDAN, S. \& SCHUSTER, K., «Evaluation of pedagogical quality in early childhood education: a cross national perspective», Journal of Research in Childhood Education, 16, 2001, p. 109-125.

SHONKOFF, J.P. \& PHILLIPS, D.A. (eds.)., «From neurons to neighbourhoods», The science of early childhood development, National Academy Press, Washington, D.C., 2000.

SLAVENAS, R., «Evaluation practices of model early childhood programs», Early Child Development and Care, 89 (1), 2005, P. 31-44.

STOVER, D., "Applying brain research in the classroom is not a nobrainer», The Education Digest, 66 (8), 2001, p. 26-29. 